\title{
The measurement of $\theta_{13}$ and $\delta$ : the role of the uncertainties on the solar and atmospheric parameters
}

\author{
D. Meloni ${ }^{\mathrm{a}}$ \\ ${ }^{a}$ INFN, Sezione di Roma e Dip. di Fisica, Univ. di Roma "La Sapienza", P.le A. Moro 2, I-00185 \\ Roma, Italy
}

In this talk we show how the errors on solar and atmospheric parameters affect the measurement of the unknown PMNS parameters $\theta_{13}$ and $\delta$ at future LBL facilities. Performing three parameters fits in $\theta_{13}, \delta$ and, in turn, one of the atmospheric or solar parameters, we show that present uncertainties on $\theta_{23}$ and $\Delta m_{23}^{2}$ worsen significantly the precision on $\left(\theta_{13}, \delta\right)$ whereas the solar sector does not introduce further uncertainties. A precision on the atmospheric parameters similar to what expected at T2K-I is necessary to improve the sensitivities to $\theta_{13}$ and $\delta$.

\section{Motivations}

The atmospheric and solar sector of the PMNS leptonic mixing matrix have been measured with quite good resolution by SK, SNO and KamLand. These experiments measure two angles, $\theta_{12}$ and $\theta_{23}$, and two mass differences, $\Delta m_{12}^{2}$ and $\Delta m_{23}^{2}$. On the other hand, only an upper bound exists on the other angle, $\theta_{13}$, and the $\mathrm{CP}$-violating phase $\delta$ is completely unknown [1. Two additional discrete unknowns are the sign of the atmospheric mass difference and the $\theta_{23}$-octant (if $\theta_{23} \neq 45^{\circ}$ ).

The strong correlations between $\theta_{13}$ and $\delta$ and the presence of parametric degeneracies in the $\left(\theta_{13}, \delta\right)$ parameter space [2] (the so-called clones), make the simultaneous measurement of the two variables extremely difficult. In the literature, this has been normally studied considering the solar and atmospheric mixing parameters as external quantities fixed to their best fit values (see for example Ref. [3] and refs. therein). However, the experimental uncertainties on these parameters can in principle affect the measurement of the unknowns, and it seems important to perform an analysis that goes beyond the two-parameters fits presented in the literature.

In this talk we therefore present the impact that "solar" (i.e. $\theta_{12}$ and $\Delta m_{12}^{2}$ ) and "atmospheric" (i.e. $\theta_{23}$ and $\Delta m_{23}^{2}$ ) parameters uncertainties have on the measurement of $\theta_{13}$ and $\delta$, studying their effects at three of the many pro- posed setups: the 4 MWatt SPL Super-Beam 4, a $\gamma \sim 100 \beta$-Beam [5] and the CERN-based $50 \mathrm{GeV}$ Neutrino Factory (considering both the "golden" 6] and "silver" 7] channels).

\section{The strategy}

With the aim of understanding how any single parameter affects the measurements of $\theta_{13}$ and $\delta$, we performe a series of three-parameters fits (taking $x=\theta_{12}, \Delta m_{12}^{2}, \theta_{23}$ and $\Delta m_{23}^{2}$ in turn as the third fitting variable) to be compared with standard two-parameters fits in $\theta_{13}$ and $\delta$. For each transition channel $\nu_{\alpha} \rightarrow \nu_{\beta}$, sign of $\Delta m_{23}^{2}$ $\left(s_{a t m}= \pm 1\right.$ for positive and negative values respectively) and octant of $\theta_{23} \quad\left(s_{\text {oct }}= \pm 1\right.$ for $\theta_{23}>$ or $<45^{\circ}$ respectively), we build a $\chi^{2}$ function :

$$
\left[\chi^{2}\left(\theta_{13}, \delta, x\right)\right]_{\alpha \beta}=\sum_{ \pm}\left[\frac{N_{\alpha \beta}^{ \pm}(\vec{g})-N_{\alpha \beta}^{ \pm}(\vec{t})}{\delta N_{\alpha \beta}^{ \pm}}\right]^{2}
$$

where $\vec{g}=\left(\theta_{13}, \delta, x ; s_{a t m}, s_{\text {oct }}\right)$ and $\vec{t}=$ $\left(\bar{\theta}_{13}, \bar{\delta}, \bar{x} ; \bar{s}_{\text {atm }}, \bar{s}_{\text {oct }}\right)$ are vectors of guessed and true (that is, chosen by Nature) parameters. \pm refers to neutrinos or antineutrinos and $N_{\alpha \beta}^{ \pm}$is the number of charged leptons $l_{\beta}^{ \pm}$observed in the detector for a $\nu_{\alpha}\left(\bar{\nu}_{\alpha}\right)$ beam. The error $\delta N_{\alpha \beta}^{ \pm}$on the sample takes into account the statistical error on $N_{\alpha \beta}^{ \pm}$as well as the sum of beam and detector backgrounds and the total systematic error (see 
8 for details). The three-parameters $\chi^{2}$ function defines a three-dimensional 90\% CL contour that is eventually projected onto the $\left(\theta_{13}, \delta\right)$ plane to perform a direct comparison with the standard two-parameters 90\% CL contours for the considered setups 9 .

\section{The results}

By comparing the results at the three, very different, facilities, we deduce that the impact of the current atmospheric parameters uncertainties is a common problem that future experiments looking for $\theta_{13}$ and $\delta$ will have to face.

Especially for larger values of $\bar{\theta}_{13}$ (and almost every value of $\bar{\delta}$ ), the present uncertainties on the atmospheric parameters are large enough to modify in a significant way the results of twoparameters fits, resulting in a lost of precision on $\theta_{13}$ and $\delta$. We have noticed that this is mainly due to the wide displacements of the clones, which are free to move in the multi-dimensional manifold to arrange for a lower $\chi^{2}$. An example at the $\beta$-Beam facility is shown in plot (a) of Fig 1 in which the output of our statistical approach (for $x=\theta_{23}$ and the true solution only, $s_{a t m}=\bar{s}_{\text {atm }}$ and $s_{\text {oct }}=\bar{s}_{\text {oct }}$ ) is compared with a usual twoparameters fit.

The situation is quite different if the precision on the atmospheric parameters is improved. This could be achieved by T2K-I experiment [10] and by the SPL Super-Beam itself. Using their expected errors on $\theta_{23}$ and $\Delta m_{23}^{2}$, we have observed that in much of the $\left(\theta_{13}, \delta\right)$ parameter space there is a general improvement on the precision of these parameters (see plot (b) in Fig (1) but some extra clones are still present in three-parameters fits that were absent in the two-parameters analysis. This is a clear indication of the fact that the problem we are addressing must be seriously taken into account when envisaging future facilities to look for $\theta_{13}$ and $\delta$, even in the case of reduced uncertainties on $\theta_{23}$ and $\Delta m_{23}^{2}$.

As a final remark, we stress that the impact of solar parameters uncertainties on the measurement of $\left(\theta_{13}, \delta\right)$ is negligible, at least above the verge of the $\theta_{13}$-sensitivity for the considered facilities.

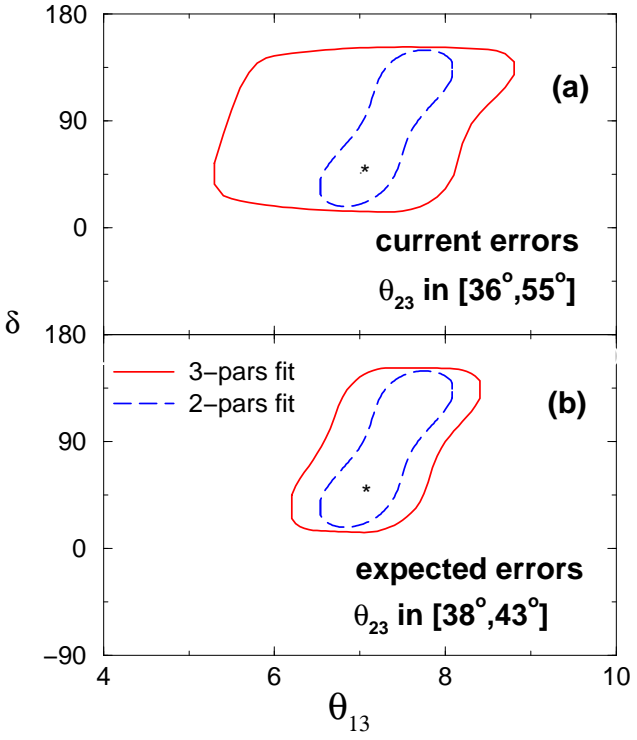

Figure 1. Comparison of the projection of threeparameters $90 \%$ CL contours onto the $\left(\theta_{13}, \delta\right)$ plane (solid lines) with the corresponding twoparameters $90 \%$ CL contours (dashed lines) after a 10 years run at the $\beta$-Beam. Star represent the projection of the input point $\left(\bar{\theta}_{13}, \bar{\delta}, \bar{\theta}_{23}\right)=\left(7^{\circ}\right.$, $\left.45^{\circ}, 40^{\circ}\right)$. See [8] for further details.

\section{REFERENCES}

1. E. Lisi, these Proceedings

2. S. Rigolin, these Proceedings

3. M. Apollonio et al., arXiv:hep-ph/0210192

4. J. J. Gomez-Cadenas et al. arXiv:hep-ph/0105297.

5. J. Bouchez, M. Lindroos and M. Mezzetto, AIP Conf. Proc. 721 (2004) 37.

6. A. Cervera et al., Nucl. Phys. B 579, 17 (2000) [Erratum-ibid. B 593, 731 (2001)].

7. A. Donini et al., Nucl. Phys. B 646 (2002) 321; J. Phys. G 29 (2003) 1865; D. Autiero et al., Eur. Phys. J. C 33 (2004) 243.

8. A. Donini et al. arXiv:hep-ph/0506100 D. Meloni, Acta Phys. Polon. B 35, 2781 (2004).

9. A. Donini et al. arXiv:hep-ph/0411402

10. Y. Itow et al., arXiv:hep-ex/0106019. 